\title{
Interventions for improving adherence to airway clearance treatment and exercise in people with cystic fibrosis (Protocol)
}

Jones M, Moffatt F, Harvey A, Ryan JM

Jones M, Moffatt F, Harvey A, Ryan JM.

Interventions for improving adherence to airway clearance treatment and exercise in people with cystic fibrosis (Protocol).

Cochrane Database of Systematic Reviews 2020, Issue 5. Art. No.: CD013610.

DOI: 10.1002/14651858.CD013610.

www.cochranelibrary.com 
TABLE OF CONTENTS

HEADER 1

ABSTRACT

BACKGROUND

OBJECTIVES

METHODS

ACKNOWLEDGEMENTS

REFERENCES

APPENDICES

HISTORY

CONTRIBUTIONS OF AUTHORS

DECLARATIONS OF INTEREST

SOURCES OF SUPPORT

NOTES 
[Intervention Protocol]

\section{Interventions for improving adherence to airway clearance treatment and exercise in people with cystic fibrosis}

Mandy Jones ${ }^{1}$, Fiona Moffatt ${ }^{2}$, Alex Harvey ${ }^{3}$, Jennifer M Ryan 4

1Centre for Research in Rehabilitation, School of Health Sciences and Social Care, Brunel University, Uxbridge, UK. 2School of Health Sciences, University of Nottingham, Nottingham, UK. ${ }^{3}$ School of Health Sciences and Social Care, Brunel University, Uxbridge, UK. 4 Institute of Environment, Health and Societies, Brunel University London, Uxbridge, UK

Contact address: Mandy Jones, mandy.jones@brunel.ac.uk.

Editorial group: Cochrane Cystic Fibrosis and Genetic Disorders Group.

Publication status and date: New, published in Issue 5, 2020.

Citation: Jones M, Moffatt F, Harvey A, Ryan JM. Interventions for improving adherence to airway clearance treatment and exercise in people with cystic fibrosis (Protocol). Cochrane Database of Systematic Reviews 2020, Issue 5. Art. No.: CD013610. DOI: 10.1002/14651858.CD013610.

Copyright @ 2020 The Cochrane Collaboration. Published by John Wiley \& Sons, Ltd.

\section{A B S T R A C T}

\section{Objectives}

This is a protocol for a Cochrane Review (intervention). The objectives are as follows:

To assess the effects of interventions to enhance adherence to airway clearance treatment and exercise therapy in people with CF and their effects on health outcomes, such as pulmonary exacerbations, exercise capacity, health-related QoL and healthcare costs. 


\section{B A C K G R O U N D}

\section{Description of the condition}

Cystic fibrosis (CF) is a life-limiting autosomal recessive genetic disorder, which affects exocrine glands (predominantly lungs and pancreas) throughout the body. A genetic mutation affecting the CF membrane trans-regulator protein (CFTR) acts on chloride channels, leading to excessive reabsorption of sodium and water across cell membranes; this produces dehydrated extracellular fluid and subsequently thick secretions (Ratjen 2009). In the lungs, airway liquid depletion impairs mucociliary transport, making thick secretions difficult to clear; these accumulate causing repeated respiratory tract infection, poor mucociliary clearance, persistent bacterial colonisation and irreversible damage (Hurt 2012). Indeed, respiratory failure is the predominant cause of morbidity and mortality in CF (Radtke 2017). Consequences of progressive respiratory disease (productive cough, haemoptysis, breathlessness and reduced exercise capacity) (Bott 2009), plus pancreatic insufficiency characterised by poor absorption of protein and fat (Ratjen 2009), with subsequent malnutrition (O'Sullivan 2009), result in a reduced quality of life (QoL) (Britto 2004; Dobbin 2005).

Physiotherapy is an integral and essential component for managing CF (Bott 2009; CF Trust 2017; NICE 2017). Physiotherapy may comprise multimodal interventions, e.g. the management of musculoskeletal conditions and stress urinary incontinence (Button 2016); however, the cornerstone of treatment (and therefore the focus of this review) involves the assessment and management of cardiovascular capacity (exercise) (Radtke 2017), and airway clearance (Sherman 2019; Wilson 2019) with or without the use of non-invasive ventilation (Moran 2017). There is significant variation in the range of physiotherapy management options for people with CF, but the treatment regimen is invariably acknowledged as burdensome for the patient and carer(s) across the course of their life. The median number of treatments per day for an adult with CF is seven, which constitutes an average daily treatment duration of between 108 min to three hours (Dodd 2000; Sawicki 2009). Consequently, given the reported time burden, non-adherence to physiotherapy is an ongoing challenge in the treatment of CF (O'Donohoe 2014). Self-reported adherence to airway clearance in adults with CF is approximately $30 \%$ (Meyers 2006), with a documented decline of up to $50 \%$ in adherence in adolescence (Arias Llorente 2008). Non-adherence to exercise (defined as planned, structured, and repetitive physical activity with the aim of improving or maintaining physical fitness (Casperson 1985)) has also been described; however, it is suggested that the perception of exercise therapy is generally viewed more positively by individuals with CF given its focus on health promotion rather than illness management (Rand 2014). Factors such as the impact on activities of daily living and social functioning, treatment burden, accidental or purposeful forgetting, no perceived benefit of and "being unpleasant" have been identified as influencing non-adherence (Arias Llorente 2008; Conway 1996; Dodd 2000; George 2010). Poor adherence to all aspects of CF treatment has been associated with an increased number of infective pulmonary exacerbations and the need for intravenous (IV) antibiotic therapy (Eakin 2011). The consequences of poor physiotherapy adherence are significant both for individuals with CF (reduced QoL, malaise and mortality) and in terms of healthcare costs (Wildman 2014).

\section{Description of the intervention}

Individuals with $\mathrm{CF}$ require an extensive, complex treatment regimen focused on medication, airway clearance techniques and exercise participation (Bott 2009; NICE 2017). Adherence is defined as "the extent to which a person's behaviour (i.e. taking medication, following a diet and/or executing lifestyle changes), corresponds with agreed recommendations from a healthcare provider" (WHO 2003). Adherence and persistence to treatment in CF varies by specific intervention; adherence is generally poorer for ACT when compared to most medication use (Myers 2006). Poor adherence to physiotherapy has been estimated to be about $60 \%$ (Bregnballe 2011); it leads to an increase in episodes of acute infection and hospital admission, which may contribute to premature death (Meyers 2006). Barriers to physiotherapy adherence in people with CF are multifactorial (Abbott 1994; Bregnballe 2011; Conway 1996; Geiss 1992; George 2010; Sawicki 2009), in particular the high time-demand of daily management is evidenced (Arias Llorente 2008; Bernard 2004; Bregnballe 2011). Although adherence to physiotherapy treatment varies between individuals, it is particularly poor in adolescents and young adults with CF (Arias Llorente 2008; Bregnballe 2011; Conway 1996; Eakin 2011; George 2010; O'Donohoe 2014). Clinical variables (e.g. disease severity), demographic characteristics and knowledge for the condition have not been demonstrated as reliable predictors of treatment adherence (Sherman 2019). Contemporary work has considered the application of social cognitive theory, in particular the influence of psychosocial variables such as self-efficacy and self-confidence in overcoming barriers to treatment components, as well as the influence of subjective norms (Bishay 2016; George 2010; Sherman 2019).

Interventions that aim to enhance adherence to physiotherapy in people with CF may improve functional and healthrelated QoL; these include psycho-educational interventions (leaflets, videos, apps aimed at increasing knowledge and understanding), psychotherapeutic or motivational interventions (cognitive behavioural therapy (CBT), contingent privileges or rewards, setting realistic expectations, motivational interviewing), or organisational interventions (self-management diary, electronic reminders, technology-based interventions such as active gaming, apps, tele-care) (Duff 2010). Evaluation of these strategies will provide evidence to inform clinical practice and improve healthcare outcomes for people with CF.

\section{How the intervention might work}

The development of complex prophylactic management and treatment options for symptomatic relief has led to an increased median predicted survival age from young childhood to over 40 years of age (Preston 2014); however, although adherence to these interventions is essential for efficacy (Eakin 2011; Sawicki 2009), adherence to physiotherapy airway clearance techniques and exercise remains lower than other treatments (Meyers 1999). Identifying patient-reported barriers to treatment adherence has been considered essential for developing approaches targeted at modifiable factors (Sherman 2019). Psycho-educational interventions target knowledge and understanding of the condition and treatment benefits and barriers; some studies have suggested that there are significant gaps in knowledge across people with CF and their families (Lonabaugh 2018), or that the information is suppressed as part of an individual's coping style (Duff 2010). Psychotherapeutic and motivational approaches are 
broadly aimed at reducing stress, improving coping styles and strategies, and exploring ambivalence or enhancing behaviour change in favour of adherence to the prescribed treatment regimen (Goldbeck 2014). Organisational interventions assist in the organisation and time management of treatments, e.g. providing active reminders or prompts, scheduling strategies, or tailoring treatments to lifestyle or life-stage, e.g. encouraging exercise through active video gaming.

\section{Why it is important to do this review}

Physiotherapy is generally considered to be an integral and essential component of the management of CF (Bott 2009; NICE 2017). The consequences of poor physiotherapy adherence may be potentially significant both for individuals with CF (repeated respiratory infection, reduced exercise tolerance, breathlessness, reduced QoL, malaise and reduced life expectancy) and in terms of healthcare costs (increased use of pharmacology, GP access and hospital admission). As such, evidence-based strategies to inform clinical practice and improve adherence to physiotherapy management are believed to be significant for improving QoL for the individual and reducing economic burden; this area of research has been raised as a priority by the James Lind Alliance Priority Cystic Fibrosis Setting Partnership (Rowbotham 2019). The need to assess the efficacy of strategies to improve adherence to physiotherapy management is of paramount importance to optimise healthcare outcomes.

\section{O B J E C T IVES}

To assess the effects of interventions to enhance adherence to airway clearance treatment and exercise therapy in people with CF and their effects on health outcomes, such as pulmonary exacerbations, exercise capacity, health-related QoL and healthcare costs.

\section{METHOD S}

\section{Criteria for considering studies for this review}

\section{Types of studies}

We plan to include randomised controlled trials (RCTs) and quasiRCTs. Observational studies, case studies and cross-over RCTs will not be eligible for inclusion.

\section{Types of participants}

Adults and children (from aged eight years upwards) with $\mathrm{CF}$; the lower limit of eight years is based on the age at which specialist units expect children with CF to manage their physiotherapy independently (with potential supervision, but not active involvement, of parents). We will include populations of either gender, any ethnicity, any stage of disease and based in either a hospital or community setting.

\section{Types of interventions}

We plan to include any intervention aimed at enhancing adherence to physiotherapy versus no intervention, usual care or another intervention aimed at enhancing adherence to physiotherapy. We will not compare adherence to two different physiotherapy regimens.
- educational (increasing knowledge and understanding);

- behavioural (CBT, contingent privileges or rewards, setting realistic expectations);

- organisational (self-management diary, electronic reminders); or

- technology (active gaming, apps, tele-care).

We plan to evaluate adherence using these interventions to one or more of the following components of physiotherapy management; cardiovascular capacity (exercise) with or without the use of noninvasive ventilation, and airway clearance with or without the use of non-invasive ventilation.

\section{Types of outcome measures}

\section{Primary outcomes}

1. Adherence

a. electronic monitoring (\% of completed treatments)

b. confirmed observed participation by parent or carer or healthcare professional (\% of completed treatments)

c. self-reported diary ( $\%$ of completed treatments)

\section{Secondary outcomes}

1. Frequency of hospitalisation for respiratory infection (requirement for non-routine IV antibiotics)

2. Frequency of non-hospitalised respiratory infection (requirement for home unplanned, rescue or non-routine IV antibiotics)

3. Lung function
a. forced expiratory volume at one second $\left(\mathrm{FEV}_{1}\right)(\mathrm{L}$ and $\%$ predicted)
b. forced vital capacity (FVC) (L and \% predicted)
c. $\mathrm{FEV}_{1} / \mathrm{FVC} \%$
d. lung clearance index (LCI)

4. QoL measures using a disease-specific tool (e.g. Cystic fibrosis Health-Related Quaility of Life questionnaire (Quittner 2005)) or a non-specific tool (SF36)

5. Adverse events (any adverse advent associated with the intervention reported by authors (e.g. haemoptysis, pneumothorax, respiratory distress, death)

6. Exercise capacity
a. shuttle walking test
b. 6-minute walk
c. step test
d. cardio-pulmonary exercise testing (CPET) and $\mathrm{VO}_{2}$ peak

We will compare data between the intervention and control group at the end of the intervention period, plus the end of intervention and any follow-up period. We will report data from three timeperiods: immediate or short term (less than one month); medium term (one to six months); and long term (greater than six months).

\section{Search methods for identification of studies}

There will be no restrictions regarding language or publication status.

Interventions may include: 


\section{Electronic searches}

The Cochrane Cystic Fibrosis and Genetic Disorders Group's Information Specialist will conduct a systematic search of the Group's Cystic Fibrosis Trials Register for relevant studies using the following terms: physiotherapy AND treatment adherence.

The Cystic Fibrosis Trials Register is compiled from electronic searches of the Cochrane Central Register of Controlled Trials (CENTRAL) (updated each new issue of the Cochrane Library), weekly searches of MEDLINE, a search of Embase to 1995 and the prospective handsearching of two journals - Pediatric Pulmonology and the Journal of Cystic Fibrosis. Unpublished work is identified by searching the abstract books of three major cystic fibrosis conferences: the International Cystic Fibrosis Conference; the European Cystic Fibrosis Conference and the North American Cystic Fibrosis Conference. For full details of all searching activities for the register, please see the relevant section of the Cochrane Cystic Fibrosis and Genetic Disorders Group's website.

We will additionally search the following electronic databases to identify published articles and additional studies for inclusion in this review.

- MEDLINE Ovid (1946 to present)

- SCOPUS Elsevier (1823 to present)

- AMED EBSCO (Allied and Complementary Medicine; 1985 to present)

- PsychINFO APA Publishing (1806 to present)

- CINAHL EBSCO (Cumulative Index to Nursing and Allied Health Literature; 1982 to present)

- LILACS (Latin American and Caribbean Health Science Information database (www.bireme.br); 1982 to present)

We will also search online trials registries:

- the WHO International Clinical Trials Registry Platform (apps.who.int/trialsearch);

- US National Institutes of Health Ongoing Trials Register ClinicalTrials.gov (www.clinicaltrials.gov);

- ISRCTN registry (www.isrctn.com);

- HSRProj (Health Services Research Projects in Progress (// hsrproject.nlm.nih.gov/).

The database search strategies can be found in the appendices (Appendix 1).

\section{Searching other resources}

We will check the bibliographies of included studies and any relevant systematic reviews identified for further references to relevant studies.

We plan to search the following conference proceedings (last 10 years where available):

- American Thoracic Society (ATS) International Conference (www.thoracic.org);

- European Respiratory Society (ERS) International Congress (www.ersnet.org);

- British Thoracic Society (summer and winter meetings) (www.brit-thoracic.org.uk);
- UK
Cystic
Fibrosis
Conference
(UKCFC)

(www.cysticfibrosis.org.uk).

\section{Data collection and analysis}

\section{Selection of studies}

Two review authors (MOJ and FM) will independently check search results for eligible studies. Initially, both authors will screen the titles and abstracts of identified studies. If it is clear from the study title or abstract that the study is not relevant or does not meet the selection criteria, we will exclude it. If eligibility is unclear, then we will retrieve the full paper and assess this; we will also retrieve full papers for all studies that appear to meet the selection criteria. Both authors will also assess non-English papers and if necessary, translate these with the assistance of a native speaker. We will resolve any disagreement through discussion and consensus. Where we do not achieve resolution, a third author (AH) will consider the paper(s) in question (Higgins 2011). We will keep a full record of decisions and their rationale. We will contact experts and organisations in the field to obtain additional information on relevant trials.

\section{Data extraction and management}

Two review authors (MOJ and FM) will independently extract data using a standardised form. We will resolve discrepancies by consensus; if we are unable to reach agreement, a third author (AH) will consider the paper.

The form will include:

- study design;

- sample size (intervention and control groups);

- country of origin;

- study population (age, gender);

- details of the intervention (educational (increasing knowledge and understanding); behavioural (CBT, contingent privileges or rewards, setting realistic expectations); organisational (selfmanagement diary, electronic reminders); or technology (active gaming, apps, tele-care)

- results for outcomes (primary and secondary outcomes as described above) and adverse events split by time point;

- risk of bias assessments.

Where papers do not present sufficient data to enter a study into the meta-analysis, we will contact study authors to request access to the missing data.

We will compare data between the intervention and control group at the end of the intervention period, plus the end of intervention and any follow-up period. We will report data from all time-periods, e.g. immediate or short term (less than one month); medium term (one to six months); and long term (greater than six months). Where studies report multiple measures, we will use data taken at the time-point closest to these thresholds. If the studies use multiple interventions we will summarise the results for outcomes separately. We will look at the effect of any intervention on the adherence to ACT and the adherence to exercise separately; we will analyse and present the comparisons of different interventions separately. 


\section{Assessment of risk of bias in included studies}

Two review authors (MOJ and FM) will independently assess the risk of bias (ROB) in included studies using the Cochrane ROB assessment tool outlined in chapter 8 of the Cochrane Handbook for Systematic Reviews of Interventions (Higgins 2017). Where we disagree, we will resolve differences through discussion and if we do not achieve resolution for the paper(s) in question, a third author $(\mathrm{AH})$ will consider the judgement. Where the quality of studies is unclear, we will attempt to contact the study authors for clarification.

We will assess ROB based on Cochrane criteria, summarising ROB for each outcome of interest within a study.

We will assess the following criteria (using yes, no or unclear judgements).

- Adequate sequence generation?

- Adequate allocation concealment?

- Adequate blinding of assessors?

- Incomplete outcome data adequately assessed?

- Free of suggestion of selective outcome reporting?

- Free of other bias?

It may be possible that therapists or clinicians will not be blinded in all studies of this kind of intervention; in this situation we will not assess these criteria, but will reflect the potential impact of incomplete blinding in the discussion of the results.

\section{Measures of treatment effect}

For continuous variables (adherence (\% of completed treatments), hospital admission (frequency and duration), non-hospitalised infection (frequency and duration), lung function tests and QoL) we will use the pooled mean difference (MD) with $95 \%$ confidence intervals $(\mathrm{Cls})$ as the measure of treatment effect. Where different studies have used a variety of measures, we will use the standardised mean difference (SMD) with $95 \% \mathrm{Cls}$ to report the pooled treatment effect. We will use the mean (and standard deviation (SD)) post-intervention for each group in the meta-analyses. Where these data are unavailable, we will use the the mean (and SD) change from baseline for each group. For dichotomous outcomes (adherence (confirmed observed participation) and reported adverse events), we will use the pooled risk ratio (RR) as the measure of treatment effect.

\section{Unit of analysis issues}

Where studies report multiple measures, we will use data taken at the time-point closest to our stated thresholds (see above). If the studies use multiple interventions, we will summarise the results for outcomes separately. We will analyse and present comparisons of different interventions separately. An included study may be designed such that investigators compare two different adherence interventions against a control group, but it is likely the two arms will have different participants; we will analyse the two interventions separately. If the participants are the same for both arms, this may result in double-counting.

The unit of analysis will be the participant; cross-over studies are not eligible for inclusion in the review.

\section{Dealing with missing data}

Where insufficient data are available to enter a study into a metaanalysis, we will contact the study authors to request access to the missing data. We will not impute missing data. Where data are missing and we can not obtain these from the study authors, we will acknowledge this as a limitation.

\section{Assessment of heterogeneity}

We will assess heterogeneity and its impact using the $\mathrm{Chi}^{2}$ test and the $I^{2}$ test (Deeks 2017). We will interpret $I^{2}$ values of approximately $30 \%$ to $75 \%$ as moderate heterogeneity and values over $75 \%$ as substantial heterogeneity. However, we will interpret the importance of observed $\mathrm{I}^{2}$ values with reference to the magnitude of effects and the strength of evidence for heterogeneity as obtained from the $\mathrm{Chi}^{2}$ test.

\section{Assessment of reporting biases}

We will report on selective outcome reporting as detailed in Assessment of risk of bias in included studies. Where sufficient data are available (i.e. at least 10 studies), we will explore possible publication bias and small study effects using funnel plots and test for funnel plot asymmetry using Egger's test.

\section{Data synthesis}

Where sufficient data are available, we will pool data for each outcome using a random-effects meta-analysis. We will conduct separate meta-analyses to examine effects of: (1) interventions to improve adherence to airway clearance treatment; and (2) interventions to improve adherence to exercise, compared to no treatment or usual care. Where studies examine effects of multiple interventions, we will include participants from each arm in separate meta-analyses. We will used a random-effects model as we expect there to be variation between studies in terms of participants and interventions. Where sufficient data are not available for a given outcome we will describe the findings narratively.

\section{Subgroup analysis and investigation of heterogeneity}

We plan to undertake a subgroup analysis according to age, i.e. children ( 8 to 12 years of age), adolescents (12 to 18 years) and adults (18 years and over), if the available data allow. Where studies include children and adolescents (e.g. 8 to 18 years) or adolescents and adults (e.g. 12 years and over), and we are unable to extract data for each age group, we will conduct separate subgroup analyses for children and adolescents, or adolescents and adults.

\section{Sensitivity analysis}

Where sufficient data are available, we will conduct sensitivity analyses on the basis of risk of bias, specifically the effect of excluding studies at overall high risk of bias. We will define overall high risk of bias as having a judgement of a high risk of bias against at least one criteria.

\section{Summary of findings and assessment of the certainty of the evidence}

The 'Summary of findings' tables will provide key information concerning the quality of evidence, the magnitude of effect of the interventions examined, and the sum of available data on all important outcomes for a given comparison (Schünemann 
2017a). This will provide patient-important outcomes for decision making by healthcare professionals and caregivers. We will use the GRADE approach to evaluate the literature and reflect the extent to which we are confident that an estimate of the effect is correct (Schünemann 2017b). We will generate a table for each comparison that is presented in the review (Appendix 2), and where data allow we will undertake a subgroup analysis based on age. The outcomes presented in each table will include, in order of priority:

1. adherence to treatment (\% completed treatments) (reported using SMD for all results combined in the medium term (from one to six months));

2. frequency of hospitalisation in the medium term (from one to six months);

3. frequency of non-hospitalised respiratory infection in the medium term (from one to six months);

4. change from baseline in exercise capacity (by either shuttle walking test, 6-minute walk, Step Test or CPET) (reported using
SMD for all results combined in the medium term (from one to six months));

5. change from baseline in QoL (using a disease-specific tool (e.g. Cystic Fibrosis Quality of Life Questionnaire) or a non-specific tool (SF36) (reported using SMD for all results combined in the medium term (from one to six months));

6. change from baseline in $\mathrm{FEV}_{1}, \%$ predicted (medium term (from one to six months);

7. change fro baseline in FVC \% predicted (medium term (from one to six months).

\section{ACK N OWLEDGEMENTS}

This project was supported by the National Institute for Health Research, via Cochrane Infrastructure funding to the Cochrane Cystic Fibrosis and Genetic Disorders Group. The views and opinions expressed therein are those of the authors and do not necessarily reflect those of the Systematic Reviews Programme, NIHR, NHS or the Department of Health. 


\section{REFERE N CE S}

\section{Additional references}

\section{Abbott 1994}

Abbott J, Dodd M, Bilton D, Webb AK. Treatment compliance in adults with cystic fibrosis. Thorax 1994; 49(2):115-20.

\section{Arias Llorente 2008}

Aria Llorente RP, Bousono Garcia C, Diaz Martin JJ. Treatment compliance in children and adults with cystic fibrosis. Journal of Cystic Fibrosis 2008; 7(5):359-67.

\section{Bernard 2004}

Bernard RS, Cohen LL. Increasing adherence to cystic fibrosis treatment; a systematic review of behavioural techniques. Pediatric Pulmonology 2004; 37(1):8-16.

\section{Bishay 2016}

Bishay L, Sawicki G. Strategies to optimise treatment adherence in adolescent patients with cystic fibrosis. Adolescent Health, Medicine and Therapeutics 2016; 7:117-24.

\section{Bott 2009}

Bott J, Blumenthal S, Buxton M, Ellum S, Falconer C, Garrod R, et al. Guidelines for the physiotherapy management of the adult, medical, spontaneously breathing patient. Thorax 2009; 64(Supp 1):1-15. [DOI: 10.1136/thx.2008.110726]

\section{Bregnballe 2011}

Bregnballe V, Ouluf Schoiotz P, Boissen KA, Pressler T, Thastum M. Barriers to adherence in adolescents and young adults with CF: a questionnaire study in young patients and their parents. Patient Preference and Adherence 2011; 5:507-15. [DOI: 10.2147/PPA.S25308]

\section{Britto 2004}

Britto MT, Kotagal UR, Chenier T, Tsevat J, Atherton H, Willmott R. Differences between adolescents' and parents' reports of health-related quality of life in cystic fibrosis. Pediatric Pulmonology 2004; 37(2):165-71.

\section{Button 2016}

Button BM, Wilson C, Dentice R, Cox NS, Middleton A, Tannenbaum E, et al. Physiotherapy for cystic fibrosis in Australia and New Zealand: a clinical practice guideline. Respirology 2016; 21(4):656-67.

\section{Casperson 1985}

Caspersen CJ, Powell KE, Christenson GM. Physical activity, exercise, and physical fitness: definitions and distinctions for health-related research. Public Health Reports 1985; 100(2):126-31.

\section{CF Trust 2017}

. Standards of care and good clinical practice for the physiotherapy management of cystic fibrosis. Third edition. April 2017. Available at www.cysticfibrosis.org.uk/the-work-wedo/clinical-care/consensus-documents 2017.

\section{Conway 1996}

Conway SP, Pond MN, Hammett T, Watson A. Compliance with treatment in adult patients with cystic fibrosis. Thorax 1996; 51(1):29-33.

\section{Deeks 2017}

Deeks JJ, Higgins JP, Altman DG, editor(s) on behalf of the Cochrane Statistical Methods Group. Chapter 9: Analysing data and undertaking meta-analysis. In: Higgins JP, Churchill R, Chandler J, Cumpston MS, editor(s), Cochrane Handbook for Systematic Reviews of Interventions version 5.2.0 (updated June 2017). The Cochrane Collaboration, 2017. Available at www.training.cochrane.org/handbook.

\section{Dobbin 2005}

Dobbin CJ, Bartlet D, Melehan K, GunStein RR, Bye PT. The effect of infective exacerbations on sleep and neurobehavioral function in cystic fibrosis. American Journal of Respiratory and Critical Care Medicine 2005; 170(1):99-104.

\section{Dodd 2000}

Dodd ME, Webb AK. Understanding non-compliance with treatment in adults with cystic fibrosis. Journal of the Royal Society of Medicine 2000; 93(Suppl 38):2-8.

\section{Duff 2010}

Duff AJ, Latchford GJ. Motivational interviewing for adherence problems in cystic fibrosis. Paediatric Pulmonology 2010; 45(3):211-20.

\section{Eakin 2011}

Eakin MN, Bilderbeck A, Boyle MP, Mogayzel PJ, Riekert KA. Longitudinal association between medication adherence and lung health in people with cystic fibrosis. Journal of Cystic Fibrosis 2011; 10(4):258-64.

\section{Geiss 1992}

Geiss SK, Hobbs SA, Hammersley-Maercklein G, Kramer JC, Henley M. Psychological factors related to perceived compliance with cystic fibrosis treatment. Journal of Clinical Psychology 1992; 48(1):99-103.

\section{George 2010}

George M, Rand-Giovannetti D, Eakin M, Borelli B, Zettler M, Riekert K. Perceptions of barriers and facilitators: selfmanagement decisions by older adolescents and adults with CF. Journal of Cystic Fibrosis 2010; 9(6):425-32.

\section{Goldbeck 2014}

Goldbeck L, Fidika A, Herle M, Quittner AL. Psychological interventions for individuals with cystic fibrosis and their families. Cochrane Database of Systematic Reviews 2014, Issue 6. [DOI: 10.1002/14651858.CD003148.pub3]

\section{Higgins 2011}

Higgins JP, Deeks JJ, . Chapter 7: Selecting studies and collecting data. In: Higgins JP, Green S, editor(s). Cochrane Handbook for Systematic Reviews of Interventions Version 
5.1.0 (updated March 2011). The Cochrane Collaboration, 2011. Available from handbook.cochrane.org.

\section{Higgins 2017}

Higgins JP, Altman DG, Sterne JA, . Chapter 8: Assessing risk of bias in included studies. In: Higgins JP, Churchill R, Chandler J, Cumpston MS, editor(s), Cochrane Handbook for Systematic Reviews of Interventions version 5.2.0 (updated June 2017). The Cochrane Collaboration, 2017. Available from www.training.cochrane.org/handbook.

\section{Hurt 2012}

Hurt K, Bilton D. Haemoptysis: diagnosis and treatment. Acute Medicine 2012; 11(1):39-45.

\section{Lonabaugh 2018}

Lonabaugh KP, O'Neal KS, McIntosh H, Condren M. Cystic fibrosis-related education: are we meeting patient and caregiver expectations? Patient Education and Counseling 2018; 101(10):1865-70

\section{Meyers 1999}

Meyers LB, Meyers F. The relationship between control beliefs and self-reported adherence in adults with cystic fibrosis. Psychology, Health and Medicine 1999; 4(4):387-92.

\section{Meyers 2006}

Meyers LB, Horn SA. Adherence to chest physiotherapy in adults with cystic fibrosis. Journal of Health Psychology 2006; 11(6):915-26

\section{Moran 2017}

Moran F, Bradley JM, Piper AJ. Non-invasive ventilation for cystic fibrosis. Cochrane Database of Systematic Reviews 2017, Issue 2. [DOI: 10.1002/14651858.CD002769.pub5]

\section{Myers 2006}

Myers LB, Horn SA. Adherence to chest physiotherapy in adults with cystic fibrosis. Journal of Health Psychology 2006; 11(6):915-26.

\section{NICE 2017}

National Institute Clinical Excellence. Cystic Fibrosis; Diagnosis and Management [NG78]. Available at www.nice.org.uk/ guidance/ng78/chapter/Recommendations 2017.

\section{O'Donohoe 2014}

O'Donohoe R, Fullen BM. Adherence of subjects with cystic fibrosis to their home programme: a systematic review. Respiratory Care 2014; 59(11):1731-46.

\section{O'Sullivan 2009}

O'Sullivan BP, Freedman SD. Cystic Fibrosis. Lancet 2009; 373(9678):1891-904.

\section{Preston 2014}

Preston W. Highlights of the 2014 patient registry data. Available at www.cff.org/our-research/CF-patient-data-registry/ highlights-of-the-2014-patient-registry-data/ 2014.

\section{Quittner 2005}

Quittner AL, Buu A, Messer MA, Modi AC, Watrous M. Development and validation of The Cystic Fibrosis Questionnaire in the United States: a health-related quality-oflife measure for cystic fibrosis. Chest 2005; 128(4):2347-54.

\section{Radtke 2017}

Radtke T, Nevitt SJ, Hebestreit H, Kriemler S. Physical exercise training for cystic fibrosis. Cochrane Database of Systematic Reviews 2017, Issue 11. [DOI: 10.1002/14651858.CD002768.pub4]

\section{Rand 2014}

Rand S, Prasad A. Exercise as part of a cystic fibrosis therapeutic routine. Expert Review of Respiratory Medicine 2012; 6(3):341-52.

\section{Ratjen 2009}

Ratjen F. Cystic Fibrosis: pathogenesis and future treatment strategies. Respiratory Care 2009; 54(5):595-605.

\section{Rowbotham 2019}

Rowbotham NJ, Smith SJ, Elliott ZC, Leighton PA, Rayner OC, Morley R, et al. Adapting the James Lind Priority Setting Process to better support patient participation: an example from cystic fibrosis. Research Involvment and Engagement 2019; 5:24. [DOI: 10.1186/s40900-019-0159-x]

\section{Sawicki 2009}

Sawicki GS, Sellers De Robinson WM. High treatment burden in adults with cystic fibrosis: challenges to disease selfmanagement. Journal of Cystic Fibrosis 2009; 8(2):91-6.

\section{Schünemann 2017a}

Schünemann HJ, Oxman AD, Higgins JP, Vist GE, Glasziou P, Akl E, et al, on behalf of the Cochrane GRADEing Methods Group and the Cochrane Statistical Methods Group. Chapter 11: Completing 'Summary of findings' tables and grading the confidence in or quality of the evidence. In: Higgins JP, Churchill R, Chandler J, Cumpston MS, editor(s), Cochrane Handbook for Systematic Reviews of Interventions version 5.2.0 (updated June 2017). The Cochrane Collaboration, 2017. Available from www.training.cochrane.org/handbook.

\section{Schünemann 2017b}

Schünemann HJ, Oxman AD, Vist GE, Higgins JP, Deeks JJ, Glasziou P, et al on behalf of the Cochrane GRADEing Methods Group and the Cochrane Statistical Methods Group. Chapter 12: Interpreting results and drawing conclusions. In: Higgins JP, Churchill R, Chandler J, Cumpston MS, editor(s), Cochrane Handbook for Systematic Reviews of Interventions version 5.2.0 (updated June 2017). The Cochrane Collaboration, 2017. Available from www.training.cochrane.org/handbook.

\section{Sherman 2019}

Sherman A, Simonton-Atchley S, Campbell D, Reddy R, O'Brien C, Guinee B, et al. Persistent adherence to airway clearance therapy in adults with cystic fibrosis. Respiratory Care 2019; 64(7):778-85 


\section{WHO 2003}

World Health Organisation. Adherence to long term therapies: evidence for action. Available at www.who.int/chp/ knowledge/ publications/adherence report/en/ 2003.

\section{Wildman 2014}

Wildman MJ, Hoo ZH. Moving cystic fibrosis care from rescue to prevention by embedding adherence measurement in routine care. Paediatric Respiratory Reviews 2014; 15(1):16-8.

\section{AP PE N DICES}

\section{Appendix 1. Additional electronic search strategies}

\section{Wilson 2019}

Wilson LM, Morrison L, Robinson KA. Airway clearance techniques for cystic fibrosis: an overview of Cochrane systematic reviews. Cochrane Database of Systematic Reviews 2019, Issue 1. [DOI: 10.1002/14651858.CD011231.pub2]

\begin{tabular}{lll}
\hline Database & Date(s) searched & Search strategy \\
\hline Scopus Elsevier & 1823 to date & 1 cystic fibrosis OR mucoviscidosis OR mucoviscidose [Article title, Abstract, Keywords] \\
& & $\begin{array}{l}\text { 2 adhere OR adherence OR adhered OR nonadherence OR persist OR persistance OR per- } \\
\text { sisted OR compliance OR comply OR complied OR noncompliance OR concordance OR } \\
\text { nonconcordance OR cooperative OR cooperation OR cooperate OR cooperated OR unco- } \\
\text { operative OR conform }\end{array}$
\end{tabular}

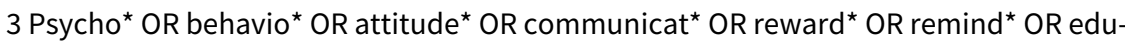
cat $^{\star}$ OR supervis ${ }^{\star}$ OR famil ${ }^{\star}$ OR train ${ }^{\star}$ OR monitor ${ }^{\star}$ OR problem ${ }^{\star}$ OR team ${ }^{\star}$ OR diar ${ }^{\star}$ OR counsel ${ }^{\star}$ OR feedback OR reinforc ${ }^{\star}$ OR motivat ${ }^{\star}$ OR self-regulat* OR cope OR coping OR emotion*

4 social $^{\star}$ OR socio* OR cognitive OR mental ${ }^{\star}$ OR verbal ${ }^{\star}$ OR condition* OR knowledge OR personal OR construct OR crisis OR aversion OR assertiv ${ }^{\star}$ OR Accept* OR commit ${ }^{\star}$ OR mind $^{\star}$ OR mood ${ }^{\star}$ OR support ${ }^{\star}$ OR well ${ }^{\star}$ OR habit* OR talk* OR depress ${ }^{\star}$

5 technology OR app* OR telehealth OR telecare OR telemedicine OR interactive OR mobile OR web OR internet OR online OR game OR games OR gaming OR text OR messag* OR telemonitoring OR chip OR chipped OR tracking OR tracker OR digital OR Bluetooth OR video

6 smartphone OR iphone OR ipad OR electronic OR computer OR reward* OR schedul*

OR telerehab*

7 \#3 OR \#4 OR \#5 OR \#6

8 physiotherap* OR exercis* OR “non-invasive ventilation” OR NIV OR "airway clearance”

9 \#1 AND \#2 AND \#7 AND \#8

*NOTE: maximum number of characters exceeded in Line \#3, so intervention search terms divided into four search lines and combined using OR operator (Line \#7)

\begin{tabular}{lll}
\hline Medline Ovid & 1946 to date & Search terms to be based on Scopus search strategy \\
\hline $\begin{array}{l}\text { PsychINFO APA } \\
\text { Publishing }\end{array}$ & 1806 to date & Search terms to be based on Scopus search strategy \\
\hline $\begin{array}{l}\text { CINHAL EBSCO } \\
\text { (Cumulative Index }\end{array}$ & 1982 to date & Search terms to be based on Scopus search strategy \\
to Nursing and Al- \\
$\begin{array}{l}\text { lied Health Litera- } \\
\text { ture) }\end{array}$
\end{tabular}


(Continued)
WHO Internation-
Search terms to be based on Scopus search strategy
al Clinical Tri-
als Registration
Platform (ICTRP)
(apps.who.int/tri- alsearch)

\begin{tabular}{lll}
\hline $\begin{array}{l}\text { AMED EBSCO (Al- } \\
\text { lied and Comple- } \\
\text { mentary Medicine) }\end{array}$ & 1985 to date & [Advanced Search] \\
& cystic fibrosis OR mucoviscidosis OR mucoviscidose [Title, abstract, subject] \\
& AND \\
& adhere OR adherence OR adhered OR nonadherence OR persist OR persistance OR per- \\
& sisted OR compliance OR comply OR complied OR noncompliance OR concordance OR \\
& nonconcordance OR cooperative OR cooperation OR cooperate OR cooperated OR unco- \\
& operative OR conform [Title, abstract, subject]
\end{tabular}

\begin{tabular}{|c|c|c|}
\hline LILACS (Latin & 1982 to date & [Advanced Search] \\
\hline $\begin{array}{l}\text { American and } \\
\text { Caribbean Health }\end{array}$ & & cystic fibrosis OR mucoviscidosis OR mucoviscidose [Title, abstract, subject] \\
\hline $\begin{array}{l}\text { Science Informa- } \\
\text { tion database }\end{array}$ & & AND \\
\hline (www.bireme.br)) & & $\begin{array}{l}\text { adhere OR adherence OR adhered OR nonadherence OR persist OR persistance OR per- } \\
\text { sisted OR compliance OR comply OR complied OR noncompliance OR concordance OR } \\
\text { nonconcordance OR cooperative OR cooperation OR cooperate OR cooperated OR unco- } \\
\text { operative OR conform [Title, abstract, subject] }\end{array}$ \\
\hline
\end{tabular}

US National Institutes of Health Ongoing Trials Register ClinicalTrials.gov (www.clinicaltrials.gov)

\author{
[Advanced Search] \\ CONDITION/ DISEASE: cystic fibrosis OR mucoviscidosis OR mucoviscidose
}

OTHER TERMS: adhere OR adherence OR adhered OR nonadherence OR persist OR persistance OR persisted OR compliance OR comply OR complied OR noncompliance OR concordance OR nonconcordance OR cooperative OR cooperation OR cooperate OR cooperated OR uncooperative OR conform

STUDY TYPE: Interventional Studies (Clinical Trials)

\section{ISRCTN registry}

(www.isrctn.com)

\section{[Basic Search]}

(cystic fibrosis OR mucoviscidosis OR mucoviscidose) AND (adhere OR adherence OR adhered OR nonadherence OR persist OR persistance OR persisted OR compliance OR comply OR complied OR noncompliance OR concordance OR nonconcordance OR cooperative OR cooperation OR cooperate OR cooperated OR uncooperative OR conform)

\section{[Basic Search]}

HSRProj (Health

Services Re-

search Projects in

Progress (https://

hsrproject.nlm.ni-

h.gov/)

\section{Appendix 2. Example summary of findings table}


Interventions to improve adherence to airway clearance treatment compared to no treatment or usual care for people with cystic fibrosis

Patient or population: people with cystic fibrosis

Settings: hospital or community

Intervention: interventions to improve adherence to airway clearance treatment

Comparison: no treatment or usual care

\begin{tabular}{|c|c|c|c|c|c|c|}
\hline \multirow[t]{3}{*}{ Outcomes } & \multicolumn{2}{|c|}{$\begin{array}{l}\text { Illustrative compara- } \\
\text { tive risks }{ }^{\star}(95 \% \mathrm{CI})\end{array}$} & \multirow{3}{*}{$\begin{array}{l}\text { Relative } \\
\text { effect } \\
(95 \% \mathrm{CI})\end{array}$} & \multirow{3}{*}{$\begin{array}{l}\text { No of par- } \\
\text { ticipants } \\
\text { (studies) }\end{array}$} & \multirow{3}{*}{$\begin{array}{l}\text { Quality of } \\
\text { the evi- } \\
\text { dence } \\
\text { (GRADE) }\end{array}$} & \multirow[t]{3}{*}{$\begin{array}{l}\text { Com- } \\
\text { ments }\end{array}$} \\
\hline & $\begin{array}{l}\text { Assumed } \\
\text { risk }\end{array}$ & $\begin{array}{l}\text { Corre- } \\
\text { sponding } \\
\text { risk }\end{array}$ & & & & \\
\hline & $\begin{array}{l}\text { Place- } \\
\text { bo/Inter- } \\
\text { vention B }\end{array}$ & $\begin{array}{l}\text { Interven- } \\
\text { tion A }\end{array}$ & & & & \\
\hline
\end{tabular}

\section{Adherence to treatment}

(\% completed treatments)

Follow-up: medium terma

\section{Frequency of hospitalisation}

Follow-up: medium terma

\section{Frequency of non-hospitalised respiratory infec-}

tion

Follow-up: medium terma

\section{Exercise capacity (change from baseline)}

Follow-up: medium terma

\section{QoL (change from baseline)}

(using a disease-specific tool or a non-specific tool)

Follow-up: medium terma

\section{FEV $_{1} \%$ predicted (change from baseline)}

Follow-up: medium terma

\section{FVC $\%$ predicted (change from baseline)}

Follow-up: medium terma

\footnotetext{
*The basis of the assumed risk and the corresponding risk is described in the comments. For lung function outcomes, absolute data was not presented in a format which could be analysed due to the crossover design of the study, therefore only analyses of percentage change from baseline were included in this review
}

Abbreviations: $\mathbf{C l}$ : confidence interval; $\mathbf{F E V}_{\mathbf{1}}$ : forced expiratory volume in 1 second; FVC: forced vital capacity; $\mathbf{Q o L}$ : quality of life. 
(Continued)

Footnotes: a medium term is over one month and up to six months

GRADE Working Group grades of evidence

High quality: further research is very unlikely to change our confidence in the estimate of effect.

Moderate quality: further research is likely to have an important impact on our confidence in the estimate of effect and may change the estimate.

Low quality: further research is very likely to have an important impact on our confidence in the estimate of effect and is likely to change the estimate.

Very low quality: we are very uncertain about the estimate.

\section{H I S T O R Y}

Protocol first published: Issue 5, 2020

\section{CONTRIBUTIONS OF AUTHORS}

\section{Roles and responsibilities}

\begin{tabular}{ll}
\hline Task & Author \\
\hline Protocol stage: draft the protocol & MJ, FM \\
\hline Review stage: select which trials to include $(2+1$ arbiter $)$ & MJ, FM with AH as 3rd author when needed \\
\hline Review stage: extract data from trials (2 people) & MJ, FM with AH as 3rd author when needed \\
\hline Review stage: enter data into RevMan & MJ, FM \\
\hline Review stage: carry out the analysis & MJ, FM with statistical help \\
\hline Review stage: interpret the analysis & MJ, FM with statistical help \\
\hline Review stage: draft the final review & MJ, FM \\
\hline Update stage: update the review & MJ, FM \\
\hline
\end{tabular}

\section{DECLARATIONS OF INTEREST}

MJ: none known.

FM: none known.

$\mathrm{AH}$ : none known.

JR: none known.

\section{SOURCES OF SUPPORT}

\section{Internal sources}

- No sources of support supplied 


\section{External sources}

- National Institute for Health Research, UK

This systematic review was supported by the National Institute for Health Research, via Cochrane Infrastructure funding to the Cochrane Cystic Fibrosis and Genetic Disorders Group.

\section{N O T E S}

This project was supported by the National Institute for Health Research, via Cochrane Infrastructure funding to the Cochrane Cystic Fibrosis and Genetic Disorders Group. The views and opinions expressed therein are those of the authors and do not necessarily reflect those of the Systematic Reviews Programme, NIHR, NHS or the Department of Health. 